\title{
Kybernetische Aspekte der Strahlenschädigung
}

\author{
JÜRGEN KIEFER \\ Institut für Biophysik, Strablenzentrum der Justus Liebig-Universität, Gießen
}

\begin{abstract}
Cybernetic aspects of radiation damage. Starting with experimentally established radiobiological facts concerning cellular effects of ionizing radiation, the differences between single cells and tissues are discussed. A new classification for "size"-controlled tissues is proposed, and the relevant mathematical formulations are outlined. It is suggested that the different sensitivities of tissues against ionizing radiation may be explained on the basis of differently operating control mechanisms. As examples, root meristems, intestinal epithelium and blood forming organs are described in more detail.
\end{abstract}

\section{EINLEITUNG}

BERgoniÉ \& TRIBONdeAu (1906, p. 985) charakterisieren die Wirkung von Röntgenstrahlen: „Röntgenstrahlen sind wirksamer bei Zellen, die eine stärkere Teilungsaktivität zeigen; die Wirksamkeit ist größer bei Zellen, die sich noch öfter teilen werden, und bei Zellen, deren Morphologie und Funktion am geringsten fixiert sind.“

Wenn das so formulierte Prinzip auch im Laufe der Zeit einige Modifikationen erfahren hat, so bildet es doch auch heute noch die Grundlage vieler Untersuchungen. Denn die meist untersuchten strahlenbiologischen Testobjekte sind Organismen oder Gewebe mit hoher reproduktiver Aktivität: Einzeller, Pflanzenwurzeln, Haut- und Darmepithel, blutbildende Organe, weil der nachhaltigste Effekt der Einwirkung ionisierende: Strahlen die Hemmung oder vollständige Unterbindung der Teilungsfähigkeit ist.

In Anlehnung an BACQ \& AlexANDER (1961) kann man Gang und Ausprägung des Strahlenschadens schematisch klassifizieren (Tab. 1).

Auf jeder Ebene können Prozesse einsetzen, die den Eintritt des Strahlenschadens verhindern oder sein Ergebnis ganz oder teilweise rückgängig machen. Es wäre wünschenswert, wenn man sich bei der Beschreibung solcher Vorgänge auf eine einheitliche Terminologie festlegen könnte; in der Tabelle ist ein solcher Vorschlag gemacht: Danach sollten alle der Schädigung entgegenwirkende Prozesse, die vor der Läsion der biologisch wichtigen Moleküle eintreten, als „physikalischer Strahlenschutz“, alle, die sich auf rein chemischer Ebene abspielen, also nicht spezifisch zellgebunden sind, als "chemischer Strahlenschutz", alle zellulären Vorgänge als „Erholung“, alle Mechanismen, die durch Zusammenwirkung vieler Zellen im Gewebeverband zustande kommen, als "Restauration" oder "Restitution" bezeichnet werden. Der etwas unscharfe Begriff 
"Reparation" sollte allgemeinen Beschreibungen vorbehalten bleiben, bei denen man sich nicht auf spezielle Mechanismen festlegen kann.

Tabelle 1

Klassifikation der Strahlenschädigung

\begin{tabular}{|c|c|c|}
\hline Ebene & Angriffspunkt & $\begin{array}{l}\text { Dem Schaden entgegenwirkende } \\
\text { Prozesse }\end{array}$ \\
\hline (1) physikalische & $\begin{array}{l}\text { Ionisationen, Anregungen, } \\
\text { Bildung freier Radikale }\end{array}$ & $\begin{array}{l}\text { physik. Strahlenschutz } \\
\text { (physical protection) }\end{array}$ \\
\hline (2) chemische & Biomoleküle & $\begin{array}{l}\text { dhem. Strahlenschutz } \\
\text { (chemical protection) }\end{array}$ \\
\hline (3) zelluläre & Zelle & Erholung (recovery) \\
\hline (4) Gewebe & Gewebe & $\begin{array}{l}\text { Restauration, Restitution } \\
\text { (restoration) }\end{array}$ \\
\hline (5) Organismus & Organismus & Reaktion des Gesamtorganismus \\
\hline
\end{tabular}

Der Hauptgegenstand dieser Abhandlung wird Ebene 4, der Beeinflussung durch Zusammenwirken von $G$ e w e b s f a k t o r e n gewidmet sein, weil hier kybernetische Überlegungen möglicherweise nutzbringend angestellt werden können. Die Ebenen 1 und 2 bleiben völlig ausgespart, Zelle und Organismus werden nur gestreift und soweit behandelt, wie es ihr Zusammenhang mit der Hauptthematik erfordert.

\section{ZELLULARE EBENE}

Nach einer Zusammenstellung von Gerber (1957) reicht die Skala der letalen Dosen von $100000 \mathrm{rad}$ bei Amöben, bis zu etwa $300 \mathrm{rad}$ beim Hund, also über einen weiten Bereich. Auch auf der zellulären Ebene ergeben sich erhebliche Unterschiede. Abbildung 1 zeigt eine typische Überlebenskurve, wie sie nach Bestrahlung mit Röntgenstrahlung erhalten wird.

Bei Betrachtung der logarithmisch auf der Ordinate aufgetragenen Uberlebensfraktion kann man einen zunächst langsamen Abfall bei kleinen Dosen, der bei höheren Dosen in einen steileren (exponentiellen) Teil übergeht, erkennen. Die genaue Form des Anfangsteils, speziell die Steigung der Nullpunktstangente, ist noch nicht geklärt. Die bisher allgemein übliche mathematische Beschreibung ist die folgende:

$$
\mathrm{p}=1-\left(1-\mathrm{e}^{-\lambda \mathrm{D}}\right)^{\mathrm{n}}
$$

Neuere Analysen scheinen jedoch zu zeigen (BENDER \& Gooch 1962, PORTER 1964, Outver 1964, Kiefer 1966b), daß die folgende Formulierung den experimentellen Befunden besser angemessen ist:

$$
\mathrm{p}=\mathrm{e}^{-\mu} \mathrm{D}\left[1-\left(1-\mathrm{e}^{-\lambda \mathrm{D}}\right)^{\mathrm{n}}\right]
$$

In dieser Gleichung bedeuten p: Überlebensfraktion, D: Dosis; $\lambda$, $\mathrm{n}$ und $\mu$ sind Parameter.

Die ersten Untersuchungen mit Einzelzellen wurden an Bakterien oder anderen 
Einzellern durchgeführt. Ihre relativ große Strahlenresistenz und physiologische Verschiedenheit ließ bei Übertragung der so gewonnenen Ergebnisse auf Vielzeller, vor allem auf Säugetiere, große Vorsicht geboten erscheinen. Erst die Entwicklung der Gewebekultur von Säugerzellen (Puck 1959) gaben der Forschung ein Werkzeug in die Hand, auch diese direkt auf der zellulären Ebene zu beobachten. Es zeigte sich,

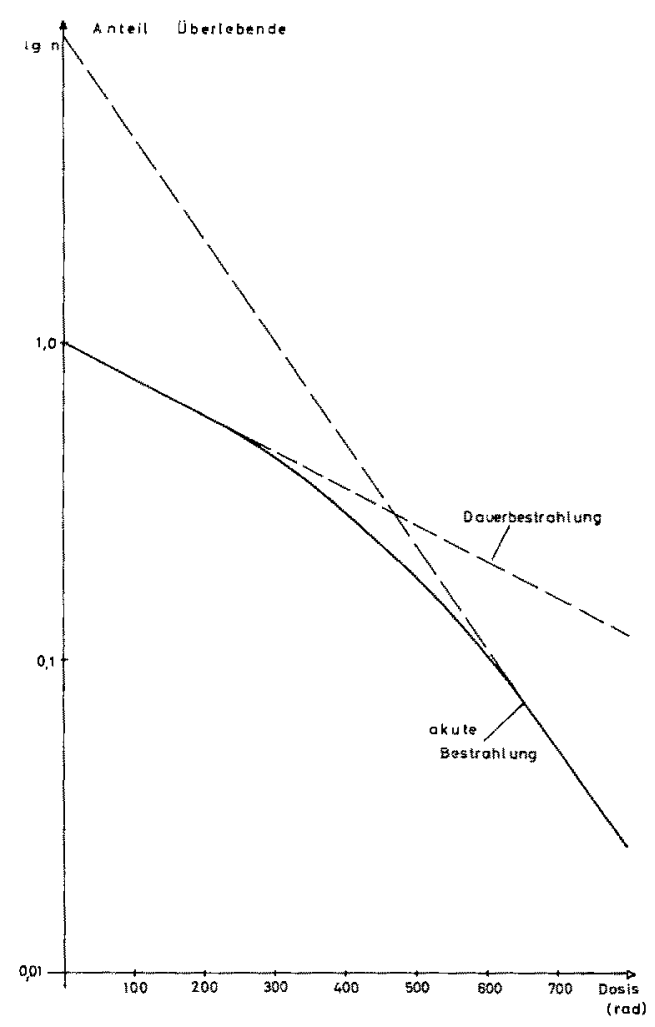

Abb. 1: Allgemeine Form der Überlebenskurven

daß ihre Empfindlichkeit um Größenordnungen höher ist als die der Bakterien, was sich durch einen entsprechend stärkeren Abfall der Uberlebenskurve bemerkbar macht. Durch die Versuche von ELKIND \& SuTton $(1959,1960)$ mittels fraktionierter Bestrahlung wurde außerdem gefunden, daß subletal geschädigte Zellen in der Lage sind, den Schaden zu reparieren, das heißt, er wird bei der Teilung nicht auf die Tochterzellen übertragen. Direkt nach dem Insult wird der Beginn der folgenden Zellteilung verzögert; die Länge dieser Verzögerungszeit ist dosisabhängig (ELKIND, HAN \& Volz 1963). Auch können letal geschädigte Zellen durchaus noch eine oder mehrere Mitosen durchlaufen. Sie verlieren nur die Fähigkeit zu unbegrenzter Proliferation. Die Ereignisse nach einem Strahleninsult bieten also ein recht komplexes Bild, wodurch Modellbetrachtungen auf Gewebsebene recht kompliziert werden und immer simplifizierende Annahmen erforderlich machen. 
Etwas einfacher liegen die Verhältnisse bei kontinuierlicher Bestrahlung. Wählt man die Dosisleistung so klein, daß die Zelle auch unter Bestrahlung subletale Schäden reparieren kann, so müßte man, wenn die Gleichung (2) als gültig angenommen wird, eine streng exponentielle Abhängigkeit der Überlebensrate von der Dosis erwarten (Oliver 1964). Sie ist auch tatsächlich an einigen Objekten gefunden worden (Berry \& COHEN 1962, Hall \& Bedford 1964, Kiefer 1966a). Über die Zyklusdauer unter Dauerbestrahlung (die Zeit $z$ wischen $z$ wei Zellteilungen) lassen sich keine allgemeinen Aussagen machen; in Krypten des Dünndarms scheint sie etwas verkürzt zu sein (FrY, Lesher, Sallese \& Staffeld 1963, Wimber \& Lamerton 1963), in Meristemzellen von Pflanzenwurzeln bei relativ hohen Dosisleistungen möglicherweise verlängert (VAN'T HofF \& SPARrow 1963a), bei anderen Untersuchungen wurde sie jedoch unverändert gefunden (VAN'T Hoff \& Sparrow 1963b, Wimber \& Lamerton 1965).

Die Zeit von einer Teilung bis zur nächsten wird als Zyklusdauer bezeichnet (im folgenden mit $\tau_{1}$ abgekürzt). Man sollte diesen Begriff nur für solche Zellen anwenden, die sich wirklich auf die nächste Teilung vorbereiten, und „ruhende" ausschließen.

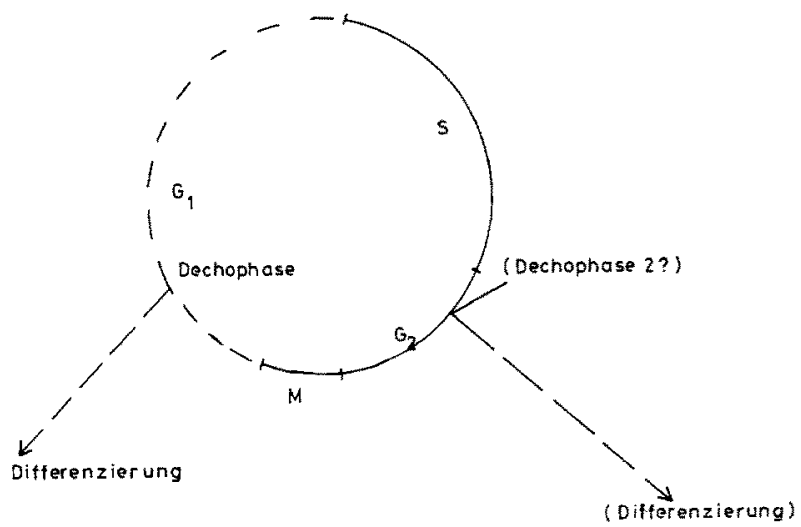

Abb, 2: Schema des Zellzyklus

Nach Howard \& PeLc (1963) teilt man den Zellzyklus in folgende Abschnitte ein: M: Mitose, $G_{1}$ : präsynthetische Phase, $S$ : Synthesephase, $G_{2}$ : postsynthetische Phase (Abb. 2). Bis auf $G_{1}$ haben alle Stadien größenordnungsmäßig eine gleiche Dauer, wenn man verschiedene Zellarten desselben Tieres vergleicht (Maurer et al. 1965). Die sehr unterschiedlichen Zyklusdauern kommen danach hauptsächlich durch die Variation der $\mathrm{G}_{1}$-Phase zustande. (Eine Ausnahme hiervon bildet möglicherweise das Hautepithel, worauf später noch eingegangen wird.) In Geweben geringer Proliferationsrate ist nur ein kleiner Anteil der Zellen wirklich im Teilungszyklus, während die anderen in $G_{1}$ verharren, bis sie zur Teilung angeregt werden. Da eine solche Ruhepause sich von der echt vorsynthetischen Periode unterscheidet, haben LAJThA, Oliver \& GURNEY (1962) vorgeschlagen, ihr die Bezeichnung " $G_{0}$ " zu geben, wodurch ein Ruhezustand im Hinblick auf die Zellteilung charakterisiert würde. Schließt man alle Zellen, auch die ruhenden, in eine summarische Berechnung der Zyklusdauer ein, dann kommt man zur "mittleren Generationszeit", die sehr starken Schwankungen unterworfen sein 
kann. Bei einem Gewebe, das nur aus proliferierenden Zellen besteht - und nur dann fallen mittlere Generationszeit und Zyklusdauer zusammen. Eine genaue Trennung der Begriffe ist auch hier dringend geboten. Die Zyklusdauer erfaßit man am besten, indem man die Zeit $z$ wischen zwei korrespondierenden Punkten zweier aufeinanderfolgenden Zyklen bestimmt, zum Beispiel durch die Beobachtung des Auftretens radioaktiv markierter Prophasen (SHERMAN, QUASTLER \& WIMBER 1961), von tetraploiden Zellen nach kurzer Colchicineinwirkung (VAN'T Hoff, WILSON \& Colon 1960) oder durch fraktionierte Colchicinbehandlung mit variablem Zeitintervall (KIEFER 1966c). Die mittlere Generationsdauer ergibt sich bei Anwendung der Technik des Metaphasenstaus mittels Colchicin (Evans, NEARY \& Tonkinson 1957).

Innerhalb jedes Zellzyklus wird mindestens ein Punkt passiert, an dem sich entscheidet, ob eine neue Teilung vorbereitet oder ein Differenzierungsprozeß begonnen wird. In den meisten Fällen verliert eine differenzierte Zelle die Teilungsfähigkeit, wenn man davon absieht, daß bei besonderem Bedarf Dedifferenzierung möglich ist. Bei der Hämatopoiese schließt auch der Differenzierungsprozeß Zellteilungen ein. Der Entscheidungspunkt wurde von Bullough (1964) „dechophase" genannt; er liegt notwendigerweise in der Ruhepause, im normalen Zyklus also wahrscheinlich in $\mathrm{G}_{1}$, im schon erwähnten Fall des Hautepithels möglicherweise auch in $G_{2}$ (Bullough 1964). Vielleicht kommen hier auch zwei Dechophasen vor.

\section{GEWEBE}

\section{Allgemeines}

In Tabelle 2 sind die mittleren Generationszeiten verschiedener Zellarten von Ratte und Maus angegeben. Abbildung 3 zeigt in schematischer Weise, innerhalb welcher Zeit sich Schädigungen einzelner Organe bei Mäusen nach Verabreichung entsprechender Einzeldosen von Röntgenstrahlen auswirken. Diese Darstellung scheint also das Prinzip von Bergonié \& Tribondeau (1906) zu bestätigen. Etwas anders sehen

Tabelle 2

Generationszeiten verschiedener Zellarten

\begin{tabular}{|c|c|c|c|}
\hline Gewebe & Tier & $\begin{array}{c}\text { Mittlere Genera- } \\
\text { tionszeit (Tage) }\end{array}$ & Literatur \\
\hline Ohr-Epidermis & Maus & 28 & LEBLOND \& WALKER (1956) \\
\hline Darmepithel & Maus & 0,75 & Maurer et al. (1965) \\
\hline Leber, Mesenchym & Maus & 7,2 & MAURER et al. (1965) \\
\hline $\begin{array}{l}\text { Niere, Tubulus- } \\
\text { epithel }\end{array}$ & Maus & 25 & MaURER et al. (1965) \\
\hline $\begin{array}{l}\text { Knochenmark } \\
\text { rote Serie }\end{array}$ & Ratte & 2,5 & LEBLOND \& WALKER $(1956)$ \\
\hline rote Serie. & Maus & 4,1 & LEBLOND \& W WALKER $(1956)$ \\
\hline weiße Serie & Ratte & 1,4 & LEBLOND \& WALKER (1956) \\
\hline weiße Serie & Maus & 6,5 & LEBLOND \& WALKER $(1956)$ \\
\hline
\end{tabular}


die Verhältnisse aus, wenn man die hierzu notwendigen Dosen betrachtet (Abb. 4). Die stark proliferierenden Zellen der Darmkrypten scheinen eine größere Resistenz zu besitzen als das Knochenmark. Noch ausgeprägter wird der Unterschied, wenn man sein Augenmerk auf die Dosisleistungen richtet, die bei Dauerbestrahlung toleriert

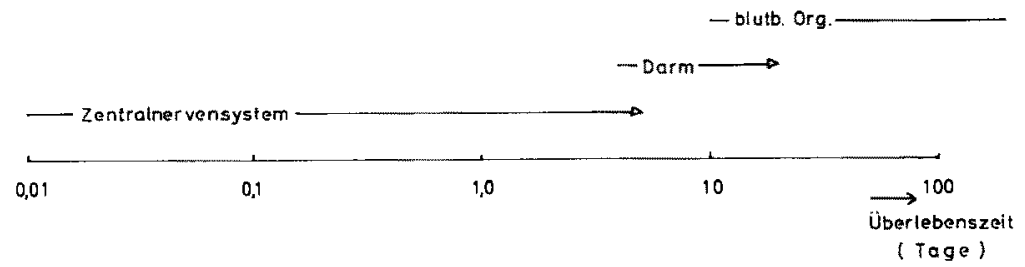

Abb. 3: Zeitdauer bis zum Eintreten des Todes durch Strahlenschädigung der hauptsächlich geschädigten Organe der Maus. (Nach Rajewsky, Aurand \& Heuss 1953)

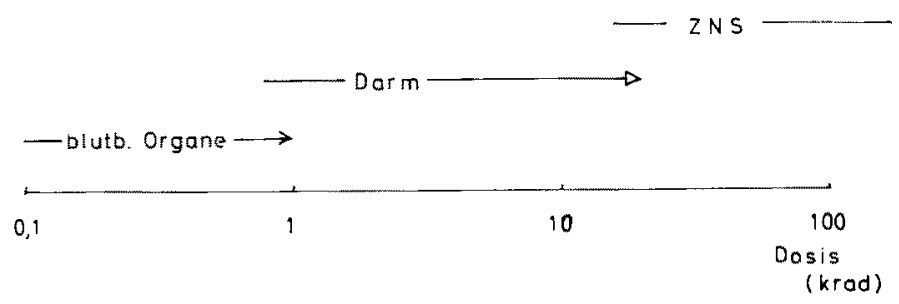

Abb. 4: Dosisbereiche, innerhalb derer die Strahlenschädigung der angegebenen Organe zum Tode führt (Maus)

werden können (LAMERTON \& LORD 1964). Von weiterem Interesse erscheint die Tatsache, daß sich bei den beiden hauptsächlich betrachteten Geweben die Strahlenschädigung unter Dauerexposition verschieden auf die Zahl der differenzierten Zellen auswirkt: Die Zahl der Zellen in den Villi des Darmes lag bei etwa 50\% des Normalwertes bei der größten tolerierbaren Dosisleistung ( $415 \mathrm{r} / \mathrm{d}$ ) (QUASTLER et al. 1959), wohingegen der Hämoglobingehalt bei der größten für das Knochenmark zu ertragenden Dosisleistung praktisch auf dem Normalwert verharrte $(84 \mathrm{r} / \mathrm{d}$; LAMERToN et al. 1960). Allerdings sank gleichzeitig die Anzahl weißer Blutkörperchen erheblich ab.

Im folgenden soll der Versuch unternommen werden, die Empfindlichkeitsunterschiede auf Grund kinetischer Betrachtungen zu deuten; dies bietet den Vorteil, daß man auf die Postulierung verschiedener Zellsensibilitäten innerhalb eines Organismus verzichten kann.

In Tabelle 3 ist eine Einteilung verschiedener möglicher Zellpopulationen vorgenommen worden, und zwar im Hinblick darauf, ob und wie homöostatische Regelungen vorkommen.

Diese Klassifikation unterscheidet sich von der durch Lajtha \& Oliver (1962) vorgeschlagenen dadurch, daß die Art der Regelung als Klassenmerkmal herangezogen wurde, und nicht die Tatsache, ob ruhende Zellen vorkommen oder nicht, also die Proliferationsrate. $\mathrm{Zu}$ der Unterteilung ist folgendes zu bemerken:

(A) Eine wirklich exponentielle Vermehrung gibt es natürlich in natura nur über 
Tabelle 3

Klassifikation von Zellpopulationstypen

\begin{tabular}{|c|c|c|c|}
\hline Typ & Bezeichnung & Charakteristikum & Beispiele \\
\hline $\begin{array}{ll}\text { A } & \\
\text { A } & 1 \\
\text { A } & 2\end{array}$ & & $\begin{array}{l}\text { ungeregelte Populationen, alle Zellen } \\
\text { teilungsfähig } \\
\text { exponentielles Wachstum } \\
\text { begrenztes Wachstum }\end{array}$ & $\begin{array}{l}\text { Chemostaten-Kultur } \\
\text { Bakterien, Tumore }\end{array}$ \\
\hline $\begin{array}{lll}\text { B } & \\
\text { B } & 1 & a \\
\text { B } & 1 & b \\
\text { B } & 2\end{array}$ & $\begin{array}{l}\text { Erneuerung- } \\
\text { systeme } \\
\text { Versorgung- } \\
\text { systeme }\end{array}$ & $\begin{array}{l}\text { geregelte Populationen, Teilung und } \\
\text { Differenzierung möglich } \\
\text { Teilung und Differenzierung direkt } \\
\text { korreliert } \\
\text { "automatische Differenzierung" } \\
\text { Teilung und Differenzierung unabhängig }\end{array}$ & $\begin{array}{l}\text { Darmepithel, Haut, } \\
\text { Leber } \\
\text { Wurzelmeristeme } \\
\text { blutbildende Organe }\end{array}$ \\
\hline $\mathrm{C}$ & & differenzierte Zellen, teilungsunfähig & \\
\hline
\end{tabular}

kurze Zeiten, in Chemostaten (Novick \& SzILARD 1950) kann sie jedoch länger aufrechterhalten werden.

(B) Die Unterscheidung zwischen Erneuerungs- und Versorgungssystemen ist zunächst rein hypothetisch. Sie liegt darin, daß für Versorgungssysteme angenommen wird, daß Zellteilung und Differenzierung nicht direkt korreliert sind: die Differenzierung setzt ein, wenn ein physiologischer Bedarf dies erforderlich macht, die Teilung, wenn die Größe des Stammzellenreservoirs unter einen bestimmten Normalwert absinkt. Teilungsrate und Differenzierungsrate sind voneinander unabhängig. Bei Erneuerungssystemen sind Differenzierungs- und Teilungsrate einander proportional mit der relativen Größe des Stammzellenreservoirs als Proportionalitätsfaktor, das heißt das Verhältnis zwischen beiden ist um so geringer, je kleiner das Stammzellenreservoir ist. Des weiteren wird angenommen, daß die Rate des turn-over bei Erneuerungssystemen von dem Mangel an gereiften Zellen abhängt.

\section{Exponentiell wachsende Zellaggregate}

Zunächst wird auf die Populationen vom Typ A eingegangen, denen keine Zellen durch Differenzierung entzogen werden. Im folgenden sind die Gleichungen zusammengestellt, mit denen eine exponentiell wachsende Population beschrieben werden kann, wobei $\mathrm{N}$ die Zellzahl und $\mathrm{K}$ die Uberlebensfraktion unter Dauerbestrahlung (pro Zellzyklus) bedeuten:

$$
\begin{aligned}
N(t) & =2 K N\left(t-\tau_{1}\right) \\
N(t) & =N_{0} e^{\frac{\ln 2 K}{\tau_{1}} t}
\end{aligned}
$$

Leider sind Untersuchungen von exponentiell wachsenden Kulturen unter Dauerbestrahlung recht selten. Eine amerikanische Dissertation (WELCH 1957), in welcher 
Hefekulturen im Chemostaten unter Bestrahlung untersucht wurden, war mir leider nicht zugänglich. Andere Autoren beobachteten die Vermehrung von Mikroorganismen unter Dauerbestrahlung von der lag-phase bis zur stationären Phase (SPOERL et al. 1954, Rubin 1954, BrüggemanN \& Giesecke 1964). Eine Deutung solcher Versuche ist schwierig, da offenbar die Strahlenresistenz von Mikroorganismen sich während des Durchlaufens der verschiedenen Wachstumsstufen ändert (STAPLeton 1955). Qualitativ läßt sich zunächst sagen, daß unter Dauerbestrahlung das Ansteigen der Zellzahl verlangsamt wird, was gleichbedeutend ist mit einer größeren mittleren Generationszeit; dabei kann die Zyklusdauer durchaus unverändert bleiben, weil nur ein gewisser Anteil der Zellen ïberhaupt zur Teilung gelangt.

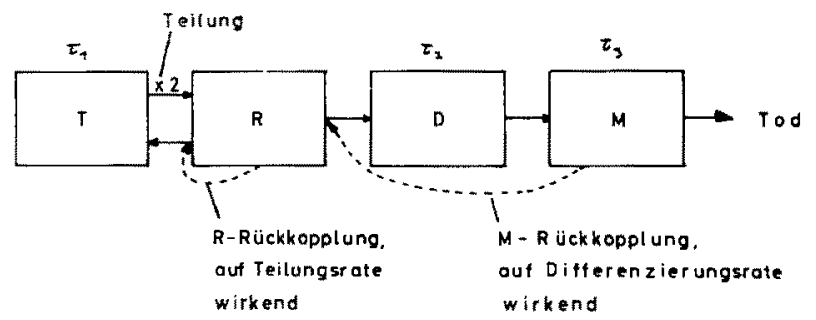

Abb. 5: Allgemeines Schema geregelter Zellpopulationen. T: Zellen im Zyklus, R: Stammzellen, D: Zellen im Differenzierungsprozeß, M: reife Zellen, $\tau_{1}$ : Zyklusdauer, $\tau_{2}$ : Dauer des Differenzierungsprozesses, $\tau_{3}$ : Lebensdauer der reifen Zellen. R-Rückkopplung: auf Teilung wirkend, M-Rückkopplung: auf Differenzierungsrate wirkend

\section{Erneuerungsgewebe}

Wenden wir uns nun den Populationen des Typs B zu: Abbildung 5 bringt ein Schema, das die Bezeichnungen klären soll: $\mathrm{R}$ ist das „Stammzellen“-Reservoir, das heißt es umfaßt alle Zellen, die teilungs- und differenzierungsfähig sind und sich weder im Zyklus noch im Differenzierungsprozeß befinden. Sie stehen also zur Teilung - um $\mathrm{R}$ zu vergrößern - als auch zur Differenzierung, zur Ergänzung der Zahl funktionsfähiger Zellen, gewissermaßen auf Abruf bereit. T bezeichnet die Gesamtheit der Zellen, die sich im Zyklus befinden, sich also auf die Teilung vorbereiten. D umfaßt alle Zellen im Differenzierungsprozeß, $M$ ist die Zahl der funktionsfähigen reifen Zellen. Die Gleichungen für Erneuerungssysteme sind die folgenden:

(a) ungestörtes System:

$$
\begin{aligned}
& \frac{d T}{d t}(t)=T^{*}(t)-T^{*}\left(t-\tau_{1}\right) \\
& \frac{d R}{d t}(t)=2 T^{*}\left(t-\tau_{1}\right)-T^{*}(t) \\
& \frac{d D}{d t}(t)=D^{*}(t)-D^{*}\left(t-\tau_{2}\right) \\
& \frac{d M}{d t}(t)=D^{*}\left(t-\tau_{2}\right)-D^{*}\left(t-\tau_{2}-\tau_{3}\right)
\end{aligned}
$$


Rückkopplungsbedingungen:

$$
\begin{aligned}
& \mathrm{D}^{*}=\frac{\mathrm{R}}{\mathrm{R}_{0}} \cdot \mathrm{T}^{*} \\
& \mathrm{D}^{*}+\mathrm{T}^{*}=\frac{1}{\tau_{1}} \cdot\left(1-\frac{\mathrm{M}}{\mathrm{M}_{0}}\right) \cdot \mathrm{R}(\mathrm{r})
\end{aligned}
$$

Fließgleichgewichte:

$$
\lim _{t \rightarrow \infty} R(t)=R_{0} \quad \lim _{t \rightarrow \infty} M(t)=M_{0} \cdot \frac{1}{1+\frac{2 M_{0} \tau_{1}}{\tau_{3} R_{0}}}
$$

(b) dauerbestrahltes System:

$$
\frac{\mathrm{dR}}{\mathrm{dt}}(\mathrm{t} / \mathrm{K})=2 \mathrm{~K} \mathrm{~T}^{* t}\left(\mathrm{t}-\tau_{1}\right)-\mathrm{T}^{*}(\mathrm{t})
$$

andere Gleichungen unverändert

Fließgleichgewichte:

$\lim R(t, K)=(2 K-1) R_{0}$

$$
\lim _{t \rightarrow \infty} M(t, K)=M_{0} \cdot \frac{1}{1+\frac{2 M_{0} \tau_{1}}{\tau_{3} R_{0}} \cdot \frac{K}{(2 K-1)^{2}}}
$$

\section{K: Uberlebensrate pro $\tau_{1}$}

Aus diesen Gleichungen ist zu folgern: (1) Das System strebt einem Fließgleichgewicht zu. Dieses ist äquifinal, das heißt es hängt nicht von den Anfangsbedingungen ab. (2) Die Turn-over-Rate, das heißt der Anteil der in $\mathrm{R}$ eintretenden und der $\mathrm{R}$ verlassenden Zellen an der Gesamtzahl, wird durch den Mangel an reifen Zellen bestimmt. Sie kann aber nicht über einen bestimmten Wert, den einmaligen Umschlag der gesamten R-Population innerhalb einer Zyklusdauer, steigen. (3) Das Verhältnis zwischen differenzierenden und sich teilenden Zellen ist eine Funktion der Größe von $R$. Bei normaler Größe von $R\left(R=R_{0}\right)$ ist die $Z a h l$ der sich teilenden und der differenzierender Zellen gleich. Hierdurch wird das Fließgleichgewicht aufrechterhalten. (4) Alle Prozesse benötigen eine bestimmte Zeit: Zwischen Aufruf zur Teilung und der Mitose liegt eine Zyklusdauer $\tau_{1}$, die Differenzierung erfordert eine Zeitspanne $\tau_{2}$, die Lebensdauer der reifen Zellen beträgt $\tau_{3}$. (5) Unter Dauerbestrahlung wird das System zerstört, wenn pro Zyklusdauer mehr als 50\% der Stammzellen sterilisiert werden. Die letale Grenze für den Organismus, in den das System als Funktionseinheit integriert ist, dürfte allerdings schon vorher erreicht sein.

Als Beispiel sollen zwei spezielle Systeme betrachtet werden, und zwar Wurzelmeristeme und die Krypten des Dünndarms. 


\section{Wurzelmeristeme}

Eine ausführliche Diskussion dieses Systems ist an anderer Stelle gegeben (KIEFER 1966a). Es unterscheidet sich von dem allgemeinen Schema und kann folgendermaßen charakterisiert werden: (1) Im unbestrahiten Meristem sind Teilungs- und Differenzie-

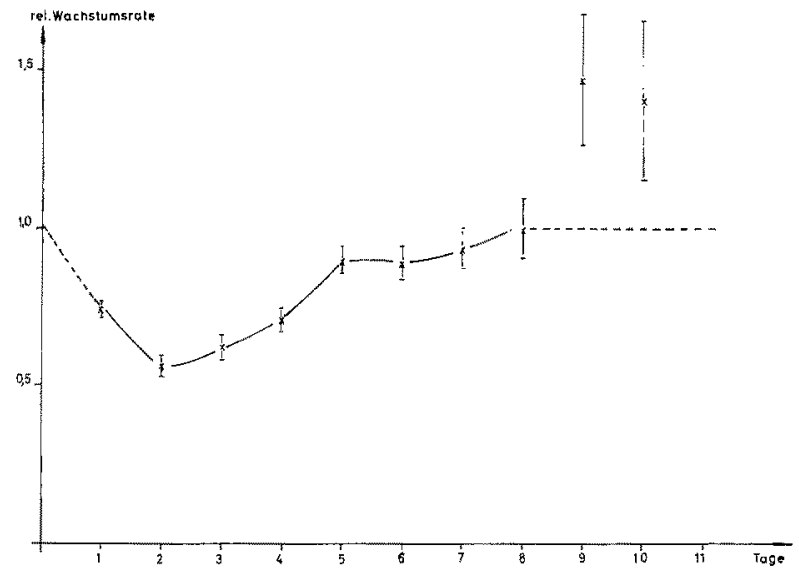

Abb. 6: Relative Wachstumsrate von Gerstenwurzeln nach $400 \mathrm{r}$ Röntgenbestrahlung zum Zeitpunkt $\mathrm{t}=0$ (bezogen auf Kontrollen gleichen Alters). (Nach KIEFER 1966b)

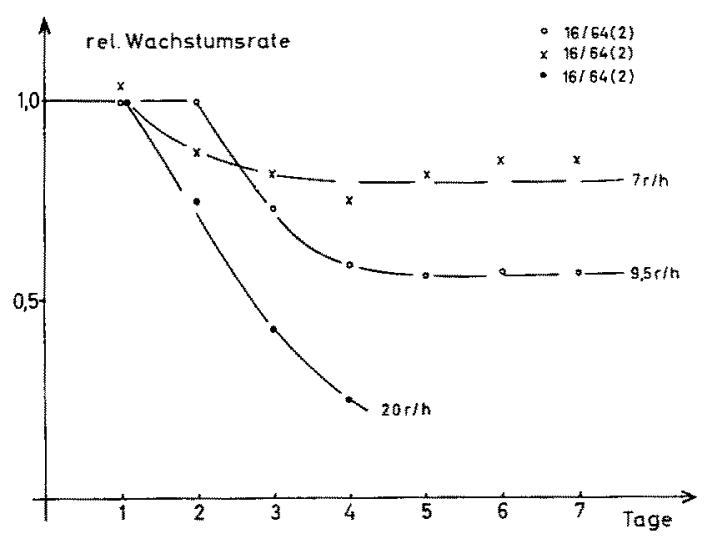

Abb. 7: Relative Wachstumsraten von Gerstenwurzeln unter Dauerbestrahlung (Röntgenstrahlen) mit verschiedener Dosisleistung. (Nach KIEFER 1966a)

rungsrate gleich (VAN'T Hoff \& YING 1964). (2) Der Anteil ruhender Zellen ist gering, sie liegen in dem sogenannten "quiescent center" (Clowes 1963). (3) Da die differenzierten Zellen in der Wurzel verbleiben, haben sie eine praktisch unendliche Lebensdauer. Man muß also annehmen, daß die Differenzierung "automatisch" erfolgt; die Turn-over-Rate ist festgelegt und liegt nahe bei $1 / \tau_{1}$. Die Abweichung von diesem Wert ergibt sich wegen der Existenz des "quiescent center“. (4) Das System kann sich 
von einer Einzelbestrahlung mit nicht zu hohen Dosen erholen, was sich darin zeigt, daß die Wachstumsrate der Wurzel zunächst abnimmt, nach Durchlaufen eines Minimums aber den Normalwert wieder erreicht (Abb. 6). (5) Unter Dauerbestrahlung stellt sich bei nicht zu hohen Dosisleistungen ein konstanter Endwert ein (Abb. 7). (6) Sowohl nach Einzel- als auch unter Dauerexposition ist die mitotische Aktivität der Zellen des "quiescent center" gesteigert (ClOwES \& HALL 1963).

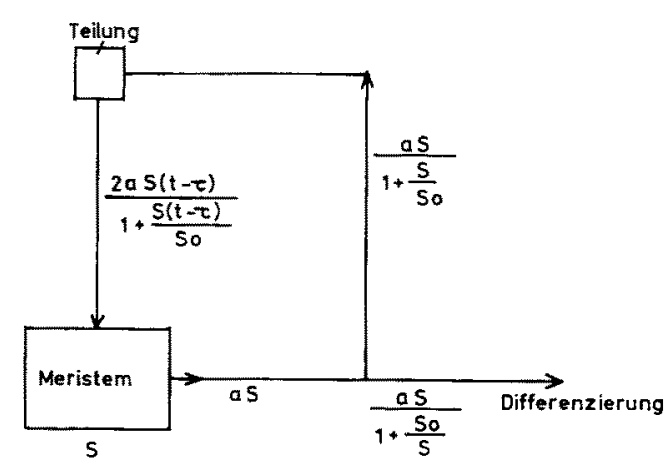

Abb. 8: Schema für die Regelung im Gerstenwurzelmeristem; S (hier): Stammzellenkompartiment, $\tau$ : Zyklusdauer. (Nach Kiefer 1966a)

Die vereinfacht auf dem Schema der Abbildung 8 beruhende Gleichung für die Regelung des Wurzelmeristems ist die folgende, wobei S: Stammzellenkompartiment und $\tau:$ Zyklusdauer bedeuten:

$$
\frac{d S}{d t}(t)=\frac{2 a S(t-\tau)}{1+\frac{S(t-\tau)}{S_{0}}}-a S(t)
$$

Zugunsten der geäußerten Vorstellung können folgende experimentelle Tatsachen herangezogen werden: (1) Die Erholungsfähigkeit nach Einzel- und das Kompensationsvermögen unter Dauerbestrahlung sprechen für das Vorliegen einer Regelung. (2) Das Modell verlangt, daß nach einem Insult der Anteil der sich teilenden Zellen steigt. Das ist zumindest für das "quiescent center" nachgewiesen. (3) Benutzt man das Modell zur Berechnung von Überlebenskurven, so ergibt sich, daß ihre Form mit derjenigen, wie sie allgemein bei strahlenbiologischen Untersuchungen gefunden werden, ubereinstimmt (KIEFER 1966a, b).

\section{Dünndarmepithel}

Ein weiteres Beispiel eines Erneuerungssystems ist das Dünndarmepithel. Auch hier sprechen experimentelle Fakten für die Plausibilität der vorgeschlagenen Hypothesen: (1) Im Normalfall sind ungefähr 50\% der Kryptenzellen im Zellzyklus (FRY et al. 1963b). Der andere Anteil befindet sich wahrscheinlich im Differenzierungsprozeß. (2) Wird die Lebensdauer der reifen Zellen verlängert, zum Beispiel durch Her- 
absetzen der Umgebungstemperatur bei Kaltblütern, so sinkt die Mitoserate in den Krypten (Hrodo 1965). (3) Nach Bestrahlung sinkt sowohl die Zahl der Kryptenzellen (W IMBer \& Lamerton 1963) als auch die der reifen Zellen in den Villi (QuASTLER et al. 1959). (4) Unter Dauerbestrahlung ist der Anteil DNS-synthetisierender Zellen leicht erhöht (WIMBER \& LAMER TON 1963). (5) Auch hier stellt sich unter Dauerexposition mit nicht zu hoher Dosisleistung ein konstanter Endwert in der Zellzahl ein (LAMERTON \& LORD 1964). (6) Unter Dauerbestrahlung ist nach anfänglichern Abfall der Mitoserate ein Ansteigen festzustellen.

Alle diese Befunde lassen sich leicht in das Populationsmodell einordnen. Eine besondere Rolle scheint das Hautepithel zu spielen, wie schon betont wurde: Nach Untersuchungen von Gelfant (1963) gibt es in ihm Zellen, die in Go verharren, also bei etwaigem Bedarf innerhalb recht kurzer Zeit zur Mitose kommen. In Ubereinstimmung hiermit sind die Ergebnisse von Rausch (1965) an Kaninchenohren, bei denen gezeigt werden konnte, daß schon zwei Stunden nach Hautläsionen Reparationsprozesse einsetzen. Diese können durch Röntgendosen gehemmt oder vollständig verhindert werden.

\section{Versorgungssysteme: Blutbildendes System}

Das spezielle Charakteristikum der Versorgungssysteme liegt daran, daß sie sich wechselnden physiologischen Anforderungen anpassen müssen. Das äußert sich daran,

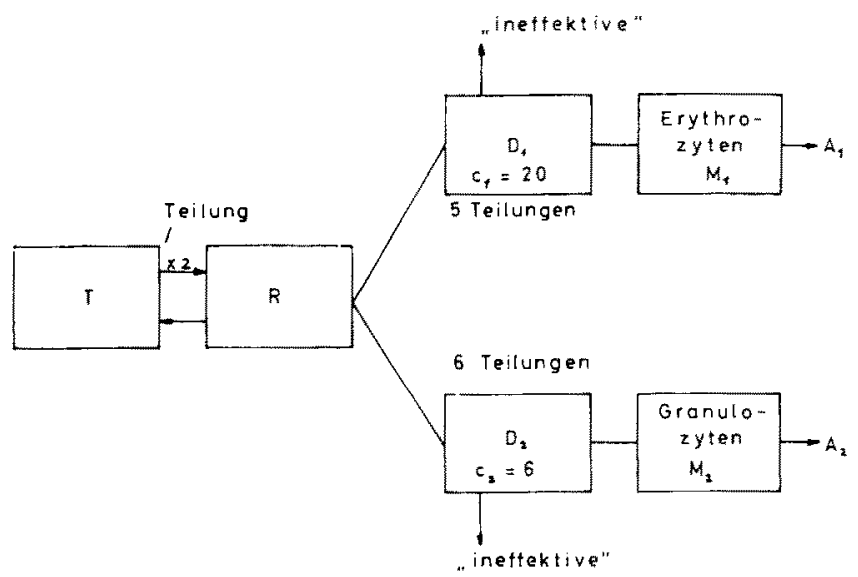

$\mathrm{Abb}, 9$ : Allgemeines Schema der Hämatopoiese

daß die Sollzahl der reifen Zellen $\mathrm{M}_{0}$ nicht konstant ist. Ebenso ist die Angabe einer Lebensdauer $\tau_{3}$ höchstens ein statistischer Mittelwert. Diese Überlegungen treffen sicher auf das granulopoietische System $\mathrm{zu}$, wahrscheinlich aber auch für die roten Blutkörperchen. Das blutbildende System ist hochkompliziert, und jede Modellbetrachtung wird nur ein sehr grobes Abbild liefern. Aus diesem Grunde soll hier das umfangreiche Schriftum nicht referiert werden (vgl. FLIEDNER \& STODTMEISTER 1962). 
Ein sehr simplifiziertes Schema ist in Abbildung 9 dargestellt. Es basiert auf Vorschlägen von Lajtha (1962) und Patt \& Maloney (1963). Aus multipotenten Stammzellen entstehen Erythrozyten und Granulozyten. Die Morphologie der Stammzellen ist bisher noch nicht eindeutig geklärt, einiges scheint dafür $\mathrm{zu}$ sprechen, daß es sich dabei um die sogenannten "kleinen Lymphozyten" des Knochenmarks handelt (CuDKowICZ et al. 1964). Durch verschiedene ingeniöse Verfahren kann über ihre Zahl Aufschluß gewonnen werden: die Milzkolonietechnik (TiLl \& McCulloch 1961), die Untersuchung der Fähigkeit, letal geschädigtes Knochenmark zu repopularisieren (Hodgson 1962) oder des Verhaltens nach Erythropoietin-Gaben (Gurney 1963). Die Differenzierungsvorgänge bei der Hämatopoiese schließen ihrerseits Zelleinteilungen ein, sind also strahlenempfindlich.

Nimmt man an, daß der Anstoß zur Differenzierung und die Tendenz, die Größe des Stammzellen-Reservoirs zu erhalten, voneinander unabhängig sind und postuliert man weiter, daß die Differenzierungsrate dem Mangel an reifen Zellen, die Teilungsrate dem Mangel an Stammzellen proportional ist, dann kommt man zu folgenden Gleichungen der Hämatopoiese:

(a) ungestörtes System:

allgemeine Gleichungen wie Erneuerungssysteme, aber mit zwei Differenzierungswegen.

$$
\text { nur: } \frac{\mathrm{d} \mathrm{M}_{\mathrm{i}}}{\mathrm{dt}}(\mathrm{t})=\mathrm{c}_{\mathrm{i}} \mathrm{D}_{\mathrm{i}}{ }\left(\mathrm{t}-\tau_{2}\right)-\mathrm{A}_{\mathrm{i}}
$$

Rückkopplungsbedingungen:

$$
\mathrm{T}^{*}=\mathrm{a}\left(1-\frac{\mathrm{R}}{\mathrm{R}_{0}}\right) \mathrm{R} \quad \mathrm{D}_{\mathrm{t}}^{*}=\mathrm{b}_{\mathrm{i}}\left(1-\frac{\mathrm{M}_{\mathrm{i}}}{\mathrm{M}_{0}}\right) \mathrm{R}
$$

Fließgleichgewichte:

$$
\begin{aligned}
& \lim _{t \rightarrow \infty} R(t)=\frac{1}{2} R_{0}\left[1+\sqrt{1-\frac{4}{a R_{0}} \cdot \Sigma \frac{A_{i}}{C_{i}}}\right] \\
& \lim _{t \rightarrow \infty} M_{K}(t)=M_{0}\left[1-\frac{a A_{K}}{2 C_{K} b_{K} Z A_{i}} 1-\left(1-\sqrt{\frac{4}{a R_{0}} \cdot \Sigma \frac{A_{i}}{C_{i}}}\right)\right.
\end{aligned}
$$

(b) dauerbestrahltes System:

$$
\begin{aligned}
& \frac{d R}{d t}(t, K)=2 K T^{*}\left(t-\tau_{1}\right)-T^{*}(t) \\
& \frac{d M_{i}}{d t}(t, K)=K_{i} D_{i}^{*}\left(t-\tau_{2 i}\right)-A_{i}
\end{aligned}
$$

Fließgleichgewichte:

$$
\lim _{t \rightarrow \infty} R(t, K)=\frac{1}{2} R\left[1-\sqrt{1-\frac{4}{a R_{0}(2 K-1)} \Sigma \frac{A_{i}}{C_{i} K_{i}}}\right]
$$


$\lim _{t \rightarrow \infty} M_{K}(t, K)=1-\frac{a A_{K}(2 K-1)}{2 b_{K} K_{K} C_{K} \Sigma \frac{A_{i}}{C_{i}}}\left[1-\sqrt{1-\frac{4}{a R_{0}(2 K-1)} \Sigma \frac{A_{i}}{C_{i} K_{i}}}\right]$

$\mathrm{K}$ : Überlebensrate pro $\tau_{1}$ der Zellen im Zyklus

$\mathrm{K}_{\mathrm{i}}$ : Uberlebensrate pro $\tau_{2}$ der Zellen im Differenzierungsprozeß

$\mathrm{C}_{\mathrm{i}}$ : Vermehrungsrate im Differenzierungsprozeß

$A_{i}$ : Verlust pro Zeiteinheit

Dabei ist vorausgesetzt, daß pro Zeiteinheit eine konstante Zahl reifer Zellen ihre Funktionsfähigkeit verliert $\left(A_{1}\right)$. In Unterschied zu den Erneuerungssystemen wird das Stammzellen-Reservoir bei erhöhter Anforderung verkleinert, bei Verminderung vergrößert. Dieser Schluß deckt sich mit dem experimentellen Befund, daß die Reaktion auf Erythropoietin-Gaben bei polyzythämischen Tieren erheblich stärker ausfällt als bei normalen (FILmanowicz \& GuRney 1961).

Die Wirkung kontinuierlicher Bestrahlung ist hier komplexer, weil auch die verschiedenen Differenzierungsstadien durch sie betroffen werden. Die maximal tolerierbare Dosisleistung hängt von der physiologischen Belastung $a b$, die sich durch die

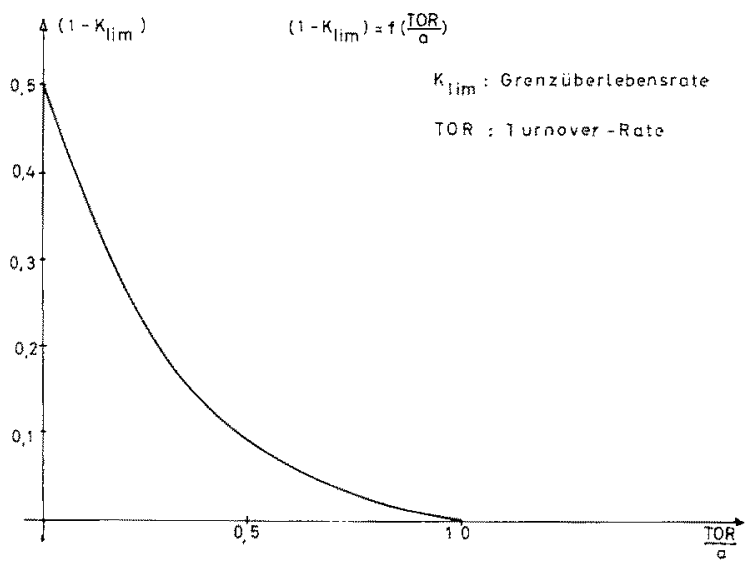

Abb. 10: Abhängigkeit der niedrigsten tolerierbaren Oberlebensrate $\mathrm{K}_{\text {lim }}$ unter Dauerbestrahlung von der Turnover-Rate des Stammzellenkompartiments im unbestrahlten Zustand (entspricht der physiologischen Aktivität)

Größen $A_{i}$ ausdrücken läßt (Gleichung 6b). Andern sich diese im Laufe des Versuchs nicht, so ist die tolerierbare Dosisleistung eine Funktion der Turnover-Rate im Stammzellen-Reservoir im unbestrahlten Zustand. Abbildung 10 zeigt die Abhängigkeit zwischen Grenzüberlebensrate $K_{\text {lim }}$ und Turnover-Rate unter der Annahme, daß die Differenzierung fünf Teilungsschritte einschließt und unter Vernachlässigung ,ineffektiver" Prozesse. $\mathrm{K}_{\lim }$ erreicht hier nur im Grenzfall 0,5 und ist sonst immer höher, das heißt, ein Versorgungssystem, wie es hier definiert wurde, ist immer empfindlicher als ein Erneuerungssystem.

Das ist aber genau das, was in den Versuchen von LAMERTON \& LORD (1964) ge- 
funden wurde. Das Darmepithel kann etwa $450 \mathrm{r} / \mathrm{d}$, das blutbildende System aber nur etwa $84 \mathrm{r} / \mathrm{d}$ tolerieren. Nimmt man an, daß die kleinen Lymphozyten wirklich die Stammzellen des Knochenmarks repräsentieren, dann läßt sich aus den Angaben von LoRd (1964) eine Turnover-Rate von ungefähr 50\% pro Zyklusdauer abschätzen. Legt man die Vorstellungen Lajthas (1962) zugrunde, so errechnet sich mit diesen Größen ein $K_{\text {lim }}=0,75$. Nimmt man weiter an, daß bei einer Dosisleistung von $500 \mathrm{r} / \mathrm{d} 50 \%$ der Zellen pro Zyklusdauer sterilisiert werden, dann kommt man für das Knochenmark auf eine tolerierbare Dosisleistung von etwa $200 \mathrm{r} / \mathrm{d}$. Bedenkt man weiterhin, daß die Zyklusdauern bei den Differenzierungssteilungen bis zu 30 Stunden (LAjtha 1962), die in den Darmkrypten etwa 12 Stunden (LAMERTon \& Lord 1964) betragen, sowie die grobe Ungenauigkeit dieser Abschätzungen, dann erscheint der Wert recht plausibel. Es ergäbe sich von hier eine Erklärungsmöglichkeit für die offensichtlichen Unterschiede in der Strahlenempfindlichkeit beider Systeme.

\section{SCHLUSSBETRACHTUNG}

Die besondere Bedeutung homöostatischer Prozesses im Organismus ist erst kürzlich von Bulıovgh \& RrtömaA (1965) behandelt worden. Es konnte gezeigt werden, daß in vielen Geweben das Fließgleichgewicht zwischen Zelltod und Erneuerung durch gewebseigene Stoffe, sogenannte Chalone, geregelt wird, die auf dem Wege über eine Hemmung der Zellteilung arbeiten. Für die Erythropoiese ist außerdem schon lange ein Stoff bekannt, der die Erythrozytenproduktion aus dem Knochenmark fördert, das Erythropoietin. Das Vorhandensein solcher Regelmechanismen muß bei Strahlenreaktionen, die über die zelluläre Ebene hinausreichen, beachtet werden; vor allem ist größte Vorsicht angezeigt bei der Extrapolation von einer Wirkungsebene auf die andere. Die allgemeine Stoffwechsellage spielt bei der Ausprägung von Strahleninsulten ebenfalls eine große Rolle. Die hier vorgelegte hypothetische mathematische Behandlung trägt diesem Sachverhalt Rechnung. Sie gibt auch eine Deutungsmöglichkeit für die Sonderstellung des blutbildenden Systems im Hinblick auf die Strahlenschädigung, indem sie eine spezielle Art der Regelung postuliert. Sie geht in ihrem Ansatz über das Knochenmarkmodell von LAjTHA et al. (1962) hinaus. Wie hier nicht im einzelnen ausgefuhrt werden kann, können so einige experimentelle Befunde, wie zum Beispiel der überschießende Einbau von ${ }^{59} \mathrm{Fe}$ nach Bestrahlung in die Modellvorstellungen mit einbezogen werden.

\section{ZUSAMMENFASSUNG}

1. Ausgehend von grundlegenden experimentellen Fakten der Strahlenbiologie werden die Unterschiede zwischen Einzelzellen und Geweben behandelt, wobei vor allem auf Regelungsvorgänge, die auf die Konstanthaltung der Zellzahl hinwirken, eingegangen wird.

2. Es wird ein allgemeines Schema für Einteilung von Zellpopulationen vorgeschlagen, 
zu welchem als Klassifizierungsmerkmal die Art und Weise homöostatischer Regelungen der Zellzahl herangezogen wird. Die Einwirkung ionisierender Strahlung auf diese Modellsysteme wird diskutiert.

3. Mit Hilfe verschiedener Annahmen wird versucht, die unterschiedliche Strahlenempfindlichkeit verschiedener Organe zu deuten.

\section{ZITIERTE LITERATUR}

Alper, T., Glules, N. E. \& Elkind, M. M., 1960. The sigmoidal curve in radiobiology. Nature, Lond. 186, 1072-1063.

Bace, Z. M. \& Alexander, P., 1961. Fundamentals of radiobiology. Pergamon pr., Oxford, $555 \mathrm{pp}$.

BENDER, M. A. \& Gooch, P. C., 1962. The kinetics of X-ray survival curves of mammalian cells in vitro. Int. J. Radiat. Biol. 5, 133-145.

Bergonié, J. \& Tribondead, L,, 1906. Interprétations de quelques resultats de la radiothérapie et essai de fixation d'une technique rationelle. C. r. hebd. Séanc. Acad. Sci., Paris $143,983-985$.

BERRY, R. J. \& COHEN, A. B., 1962. Some observations on the reproductive capacity of mammalian cells exposed in vivo to gamma-radiation at low dose-rates. Br. J. Radiol. 37, 489-491.

BrÜGgemanN, J. \& Gresecke, D., 1964. Untersuchungen über das Wachstum und die Vermehrung von Streptococcus bovis bei fortlaufender Röntgenbestrahlung. Zentbl. Bakt. Parasit Kde 192, 39-53.

Bullough, W. S., 1964. Growth regulation by tissue specific factors, or chalones. In: Cellular control mechanisms and cancer. Ed. by P. Emmelot \& O. Mühlbock. Amsterdam, Elsevier, $124-145$.

- \& RytömaA, T., 1965. Mitotic homeostasis. Nature, Lond. 205, 573-578.

Clowes, F. A. I., 1963. The quiescent centre in meristems and its behavior after irradiation. Brookhaven Symp. Biol. 16, 46-56.

- \& Halt, E. J., 1963. The quiescent centre in root meristems of Vicia faba and its behaviour after acute X-irradiation and chronic gamma-irradiation. Radiat. Bot. 3, 45-53.

Cudrowicz, G., Upton, A. C., Smith, L. H., Gosslee, D. G. \& Hughes, W. L., 1964. An approach to the characterization of stem cells in mouse bone marrow. Ann. N. Y. Acad. Sci. 114, 571-582.

Elkind, M. M., HaN, A. \& Volz, K. W., 1963. Radiation response of mammalian cells grown in culture. 4. Dose dependence of division delay and post-irradiation growth of surviving and non-surviving Chinese hamster cells. J. natn. Cancer Inst. 30, 705-721.

- \& Sutron, H., 1959. X-ray damage and recovery in mammalian cells in culture. Nature, Lond. 184, 1293-1295.

- - 1960. Radiation response of mammalian cells in culture. 1. Repair of X-ray damage in surviving Chinese hamster cells. Radiat. Res. 13, 556-593.

Evans, H. J., Neary, G. J. \& Tonkinson, S. M., 1957. The use of colchicine as an indicator of mitotic rate in broad bean root meristems. J. Genet. 55, 487-502.

Filmanowicz, E. \& Gurney, G. W., 1961. Studies on erythropoiesis. 16. Response to a single dose of erythropoietin in polycythemic mouse. J. Lab. clin. Med. 57, 65-72.

FundNer, T. M. \& Stodtmeister, R., 1962. Experimentelle und klinische Strahlenhämatologie. Lehmann, München, $80 \mathrm{pp}$.

Fry, R. J. M., Lesher, S., Kisieleski, W. E. \& Sacher, G., 1963. Cell proliferation in the small intestine. $/ n$ : Cell proliferation. Ed. by L. F. Lamerton \& R. J. M. Fry. Blackwell, Oxford, 213-233.

- - Sallese, A. \& Staffeldt, E., 1963. The generation cycle of duodenal crypt cells of mice exposed to $220 \mathrm{R}$ of Co-60 gamma-irradiation per day. Radiat. Res. 19, 628-635. 
Gelfant, S., 1963. A new theory of cell division. In: Cell growth and cell division. Ed. by R. J. C. Harris. Acad. pr., New York, 229-259. (Symp. int. Soc. Cell Biol. 2.)

Gerber, G., 1957. Ganzkörper und Teilkörperbestrahlung. In: Wissenschaftliche Grundlagen des Strahlenschutzes. Hrsg. von B. Rajewsky. G. Braun, Karlsruhe, 149-162.

Gurney, C. W., 1963. Effect of radiation on the mouse stem cell compartment in vivo, Perspect. Biol. Med. 6, 233-245.

HALL, E. J. \& BEDFord, J. S., 1964. Dose rate: its effect on the survival of HeLa cells irradiated with gamma-rays. Radiat. Res. 22, 305-315.

Hodgson, G. S., 1962. Erythrozyte Fe-59 uptake as a function of bone marrow dose injected in lethally irradiated mice. Blood 19, 460-467.

Howard, A. \& PELC, S. R, 1953. Synthesis of DNA in normal and irradiated cells and its relation to chromosome breakage. Heredity, Lond. (Suppl.) 6, 261-274.

Hrono, Y., 1965. Effects of X-irradiation on the intestinal epithelium of the goldfish, Carassius auratos. 2. Influence of temperature on the development of histopathological changes in the intestine. Radiat. Res. 24, 133-141.

KIEFER, J., 1966a. Radiation effects on barley roots. 1. Continuous exposure. Int. J. Radiat. Biol, 10, 379-390.

- 1966b. Radiation effects on barley roots. 2. Single and fractionated exposure. Int. J. Radiat. Biol. (in press).

- 1966c. A macroscopic method for the determination of cell cycle times. (In Vorbereitung)

LajтHA, L. G., 1962. Stem cell kinetics and erythropoietin. In: Erythropoiesis. Ed. by L. O. Jacobson \& M. Doyle. Grune \& Stratton, London, 140-150.

- \& Oliver, R., 1962. Cell population kinetics following different regimes of irradiation. Br. J. Radiol. 35, 131-140.

- - \& Gurney, 1962. Kinetic model of a bone-marrow stem cell population. Br. I. Haemat. 8, $442-460$.

LAMERTon, L. \& LoRd, B. I., 1964. Studies of cell proliferation under continuous irradiation. Natn. Cancer Inst. Monogr. 14, 185-197.

- Pontrfex, A. H., Blackett, N. M. \& Adams, A., 1960. Effects of protracted irradiation on the blood forming organs of the rat. 1. Continuous exposure. Br. J. Radiol. 33, 287-301.

Leblond, C. P. \& Walker, B. E., 1956. Renewal of cell populations. Physiol. Rev. 36, 255-276.

LORD, B. I., 1964. The effects of continuous irradiation on cell proliferation in rat bone marrow. Br. J. Haemat. 10, 496-507.

Maurer, W., Pilgrim, C., Wegener, K., Hollweg, S. \& Lennartz, J., 1965. Messung der Dauer der DNS-Verdopplungszeit und der Generationszeit bei verschiedenen Zellarten von Maus und Ratte durch Doppelmarkierung mit H-3 und C-14-Thymidin. Strablentherapie 60 (Sonderbd), 96-107.

Novick, A. \& SzILARd, L., 1950. Description of the chemostat. Science, N. Y. 112, 715.

Oliver, R., 1964. A comparison of the effects of acute and protracted gamma-irradiation on the growth of seedlings of Vicia faba. 2. Theoretical calculations. Int. J. Radiat. Biol. 8, 475-488.

Patt, H. M. \& Maloney, M. A., 1963. An evaluation of granulocytopoiesis. In: Cell proliferation. Ed. by L. F. Lamerton \& R. J. M. Fry. Blackwell, Oxford, 157-171.

Porter, E. H., 1964. Electronic computers and survival curves. Br. J. Radiol. 37, 610-615.

Puck, T. T., 1959. Quantitative studies of mammalian cells in vitro. Rev, mod. Phys. 31, 433-448.

Quastler, H., Bensted, J. P. M., Lamerton, L. F. \& Simpson, S. M., 1959. Adaptation to continuous irradiation: observations on the rat intestine. Br. J. Radiol. 43, 501-512.

RajeWsky, B., Aurand, K. \& Heuss, O., 1953. Weitere Untersuchungen zum Problem der Bestrahlung der weißen Maus mit hohen Dosen von Röntgenstrahlen. Z. Naturf. 8b, 524-526.

Rausch, L., 1965. Schnell ablaufende Erholungsvorgänge in der Haut nach Röntgenbestrahlung. Strablentherapie 127, 393-404.

Rubin, B. A., 1954. Growth and mutation of bacteria during continuous irradation. J. Bact. 67, $361-368$. 
Shermann, F. G., Quastler, H. \& Wimber, D. R., 1961. Cell population kinetics in the ear epidermis of mice. Expl Cell Res. 25, 114-119.

Spoerl, E., Loveless, L. E., Weisman, T. H. \& Balske, R. J., 1954. Studies on cell division. 2. X-radiation as a division inhibiting agent. J. Bact. 67, 394-401.

Stapleton, G. E., 1955. Variations in the sensitivity of $E$. coli to ionizing radiation during the growth cycle. J. Bact. 70,357-362.

Till, J. E. \& MCCulloch, E. A., 1961. A direct measurement of the radiation sensitivity of normal mouse bone marrow cells. Radiat. Res. 14, 213-222.

Van't Hof, J. \& Sparrow, A. H., 1963a. The effect of mitotic cycle duration on chromosome breakage in meristematic cells of Pisum sativum. Proc. natn. Acad. Sci. U.S.A. 50, $855-860$.

- - 1963b. Growth inhibition, mitotic cycle time and cell number in chronically irradiated root meristems of Pistm. Radiat. Bot. 3, 239-247.

- Wirson, G. B. \& Colon, A., 1960. Studies on the control of mitotic activity. The use of colchicine in the tagging of a synchronous populations of cells in the meristem of Pisum sativum. Chromosoma 11, 313-321.

- \& Y YNG, H. K., 1964. Simultaneous marking of cells in two different segments of the mitotic cycle. Nature, Lond. 202, 981-983.

WELCH, G. P., 1957. Effects of chronic exposure to X-rays on a steady rate population (of Sacharomyces cerevisiae). Thesis, Univ. of California, Berkeley.

Wimber, D. R. \& LAMERTON, L. F., 1963. Cell population studies of continuously irradiated rats. Radiat. Res. 18, 137-146.

- - 1965. Cell cycle of mouse embryonic tissue under continuously gamma-irradiation. Nature, Lond. 207, 432-433.

\section{Diskussion im Anschluß an den Vortrag KIEFER}

Scharf: Ich möchte Herrn KIefer empfehlen, sich mit Herrn Peters (Marburg) zu verständigen, der über große Mengen einschlägigen Materials verfügt (vgl. Z. woiss. Mikrosk. 62, 348, 1955; Acta bepat. 4, 1, 1956; Verh. anat. Ges., Jena 53, 49, 1957). Die moderne, chemisch orientierte Karyologie verwendet den Begriff "Ruhekern" nur noch aus Traditionsgebundenheit; der Morphologe weiß durchaus, daß der „Ruhekern “ nur eine scheinbare Ruhe repräsentiert. Sind Sie bei Ihren Ansätzen von Markofr-Prozessen (vgl. LaHres, H.: Einführung in die diskreten Markofr-Prozesse und ihre Anwendungen. Vieweg, Braunschweig, 1964) oder deren Vereinfachung ausgegangen?

KIEFER: Ich bin nicht von Markofr-Prozessen ausgegangen. Die Zellen sind als Einheiten eines Systems angesehen worden und in der einfachen Behandlung nicht enthalten. Zellzyklusdauer und Zellempfindlichkeit sind statistische Größen, die man nicht bei allen Zellen gleich ansetzen kann. Unsere Versuche sind mathematisch ohnehin schon kompliziert genug; vor allem bereiten die Zeitkonstanten Schwierigkeiten. Es gibt durchaus Zellen, beispielsweise Stammzellen des Knodhenmarks, die sich in bezug auf die Teilung in einem Ruhezustand befinden.

PANY: Der Strahlenschutz geht doch unzweifelhaft auf der Zellebene vor sich?

KIEFER: Ich wollte selbstverständlich nicht sagen, daß alles, was ich aufgezeichnet habe, isoliert yon der Zelle passiert, sondern lediglich die Ebenen, in denen diese Wirkungen zum Tragen kommen, auseinanderhalten. 